Pak. J. Agri. Sci., Vol. 55(1), 143-150;2018

ISSN (Print) 0552-9034, ISSN (Online) 2076-0906

DOI: $10.21162 / P A K J A S / 18.3572$

http://www.pakjas.com.pk

\title{
TEMPORAL STATUS OF BT GENE EXPRESSION IN PAKISTANI COTTON
}

\author{
Shaukat Ali ${ }^{1 *}$, Aamir Rana ${ }^{1}$, Muhammad Amir Zia' ${ }^{1}$, Siffat Ullah Khan ${ }^{1}$, Arshad Iqbal ${ }^{1}$ and Ghulam \\ Muhammad Ali ${ }^{1}$ \\ ${ }^{1}$ National Institute for Genomics and Advanced Biotechnology (NIGAB), National Agricultural Research Centre \\ (NARC), Park Road, 45500, Islamabad, Pakistan, ${ }^{2}$ Pakistan Central Cotton Committee, Multan, Pakistan \\ *Corresponding author's email: shaukat_parc@yahoo.co.in
}

\begin{abstract}
The present research study was conducted during 2009, 2012, 2013 and 2014 under National Cotton Varietal Trial (NCVT) for the expression profiling of $B t$ gene in local cotton genotypes/entries. Firstly, a total of 180 cotton lines (12, 75, 49 and 44 in years 2009, 2012, 2013 and 2014, respectively) were used to check the types of $B t$ gene(s) through immunostrip assay. Most of the entries were positive for the presence of CrylAc gene (Bollgard I) except CEMB-01 in 2009, DNH-105, BH-176 and CIM-573 (non Bt. Standard) in 2012, CIM-573 (non Bt. Standard) and CRIS-342 (non Bt. Standard) in 2013 and BH-185 and SLH-8 in 2014. Only a single entry (Kissan Early in 2012) was found positive for both CrylAc and Cry2Ab (Bollgard II). None of the entry gave positive result for Cry $1 F$ (Wide Strike event) through immunostrip assay. Later, expression level of CrylAc gene for all the entries were quantified through sandwich-ELISA. The expression level for twelve entries during 2009 was recorded in the range of $0.21-1.31 \mu \mathrm{g} / \mathrm{g}$ of fresh weight leaf tissue. During 2012, the expression level for CrylAc was measured in the range of $0.05-0.86 \mu \mathrm{g} / \mathrm{g}$. All the positive entries for CrylAc gave the expression level less than as recommended by EPA-

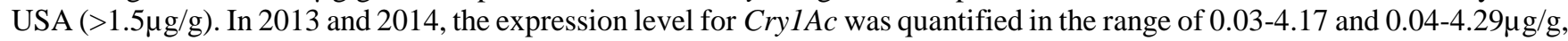
respectively. Data of four years revealed that considerable gradual improvement has been made in the levels of $B t$ toxin in local candidate cotton lines at breeder level. There is need to sustain the attained toxin levels under farmer field conditions.

Keywords: Cotton, gene expression, CrylAc, immunostrip assay, ELISA
\end{abstract}

\section{INTRODUCTION}

Cotton is the backbone of agriculture in Pakistan and is a major source of income for the farming community and textile industries. Among top five, Pakistan is the $4^{\text {th }}$ largest cotton producing country (Ali et al., 2010). To date, it is the only Genetically modified (GM) crop that has been approved for commercial cultivation in Pakistan. During the crop year 2013-14, more than 2.8 million hectares (ha) were under $B t$ cotton cultivation, utilizing $16 \mathrm{Bt}$ cotton varieties (Vasquez and Rehman, 2013). The performance of $B t$ cotton is directly related to the continuous expression of $B t$ gene(s) in the local cultivars that can vary throughout the growing season and are not consistent with the whole plant life cycle (Olsen et al., 2005; Gutierrez et al., 2006). Bt cotton is cultivated on commercial scale in many countries, but it is observed that it acts variably in toxin efficiency against target insects under different testing conditions.

The expression of $B t$ gene(s) varies according to the cotton varieties, age of plant, different parts of plant, type of $B t$ gene(s) and also its position in the genome. Further investigation highlighted that the non-uniform expression level of $B t$ gene(s) in different parts of the plant leads to the spatial variability in survival and development of lepidopterans (Adamczyk et al., 2001). Bt cotton is mostly cultivated in Sindh and Punjab provinces of Pakistan where it faces different growing conditions. Bt protein contents are mainly influenced by abiotic stresses in genetically modified cotton. For example, water logging, salinity, drought, high temperature, high level of $\mathrm{CO}_{2}$ and nitrogen deficiency can significantly reduce the insecticidal protein contents (Benedict et al., 1996; Coviella et al., 2000; Coviella et al., 2002; Barrett-Lennard, 2003; Chen et al., 2005; Iqbal et al., 2013). Bt cotton provides an efficient tool for controlling cotton bollworm, Helicoverpa armigera Hübner (Lepidoptera: Noctuidae), the major insect pest of cotton. This results in very positive economic returns to growers and reductions in insecticide use in cotton (Pray et al., 2001; Wu $\&$ Guo, 2005). However, due to the continuous production of low $B t$ toxin in engineered cotton plants, the pest could evolve resistance and nullify the benefits of $B t$ cotton (Tabashnik et al., 2008). However, the rate of resistance evolution in an insect population to a $B t$ crop depends on a number of factors, including pest population dynamics, initial frequency of resistance alleles in the pest population, genetic mode and stability of resistance, fitness of resistant individuals, temporal and spatial distribution of the insect pest on different host plants, and gene flow among different geographical populations (McGaughey and Whalon, 1992; Tabashnik, 1994; Alstad and Andow, 1995; Wu and Guo, 1997; Peck et al., 1999; Wu et al., 1999).

Since the release of $B t$ cotton in Pakistan in the year 2010, low toxin level has been remained a question mark for local cotton varieties. The initial release of varieties was allowed without 
taking care of toxin level to fill the gap. The vacuum was in fact, created due to delayed development of necessary infrastructure required for the legal release of GM crops. The year 2014 is the $5^{\text {th }}$ year of commercial release of GM cotton in Pakistan and about $30 \mathrm{Bt}$ varieties have been approved legally since 2010. The current study reports the $B t$ toxin status of local cotton varieties/lines contributed by National Cotton System in National Cotton Varietal Trials (conducted by PCCC) during four years at National Institute for Genomics and Advanced Biotechnology, Islamabad. This will help to understand the current scenario of toxin level in local $B t$ cotton varieties and answer the question and critics related to toxin level.

\section{MATERIALS AND METHODS}

Plant materials and experimental design: During 2009, 2012, 2013 and 2014, the seeds of 12, 75, 49 and 44 respectively, candidate cotton lines were provided by Pakistan Central Cotton Committee (PCCC). The soil was prepared before filling into the pots. Mixture of soil, farm yard manure and sand in the ratio of $2: 1: 1$ was fully ground and sieved. Each pot was prepared by the same ratio of mixture (soil, farm yard manure and sand). The plant material was sown in transgenic containment (Biosafety Level-II) at National Institute for Genomics and Advanced Biotechnology (NIGAB), where temperature was maintained between 25$30^{\circ} \mathrm{C}$ under natural sunlight during summer weather. Five seeds were sown in each pot $(38 \mathrm{~cm}$ in diameter and $40 \mathrm{~cm}$ in height, filled with $15 \mathrm{~kg}$ of prepared soil. Other requirements of irrigation, fertilization were maintained uniformly per pot. Immunostrip assay: Approximately 100mg fresh leaf tissue of each genotype was collected for immunostrip assay at 40 days after sowing. Samples were prepared as per manufacturer's instructions (Agdia Inc. USA) and were tested for the detection of Cry protein(s) CrylAc, Cry $2 A b$, and Cry $1 F$. Strips were carefully inserted into micro tube with prepared extract to avoid their entrance more than $0.5 \mathrm{~cm}$ during the reaction time. The appearance of control line with in 3 minutes during reaction was considered as valid. On the appearance of control line with in due time results were considered as positive $(+)$.

Sandwich-Enzyme Linked Immunosorbent Assay: After 80 days of sowing, quantification of CrylAc protein (Bt toxin) in all the entries under experimentation was carried out by sandwich-ELISA. Third fully expanded leaf tissues of each entry were used for analysis. Sandwich-ELISA was performed according to the manufacturer's instructions (Envirologix Inc. USA kit for Quantitative Elisa). The reading for optical density was measured on highly sophisticated ELISA plate reader (Bio-Rad imark ${ }^{\mathrm{TM}} \mathrm{USA}$ ) at $450 \mathrm{~nm}$. Three reading for each sample were taken and averaged. Toxin level $(\mu \mathrm{g} / \mathrm{g})$ was finally calculated by simple regression analysis using Microsoft Excel software. Finally, Bt toxin level was compared with standard one $(1.5 \mu \mathrm{g} / \mathrm{g}$ as recommended by EPA-USA).

\section{RESULTS}

Immunostrip assay: During the years 2009, 2012, 2013 and 2014 respectively, total of one hundred and eighty (180) local transgenic cotton genotypes were analyzed by immunostrip assay for determination of the types of commercial $B t$ genes (CrylAc, Cry $2 A b$ and $C r y l F)$. Results in Table-1 revealed that out of 180 genotypes, 172 genotypes harbored CrylAc gene only. Only single entry named as Kissan Early (under NCVT-2012) was positive for both CrylAc and Cry $2 A b$ (Bollgard-II event). While immunostrip test for CrylF (Wide Strike event) was $100 \%$ negative for all entries (180).

Quantification of Bt toxin (Cry1Ac) level in 12 genotypes during 2009: In 2009, twelve genotypes were used for experimentation to find the expression level of $B t$ gene(s) under National Cotton Varietal Trial (NCVT) at NIGAB. Among twelve entries, eleven genotypes were positive for CrylAc. In all positive genotypes, the expression of CrylAc gene was measured in the range of $0.21-1.31 \mu \mathrm{g} / \mathrm{g}$. The maximum expression level of CrylAc was recorded in GN-31 and $\mathrm{GN}-2085(0.88$ and $1.31 \mu \mathrm{g} / \mathrm{g}$ respectively), while the lowest expression level was measured in FH-113 $(0.21 \mu \mathrm{g} / \mathrm{g})$. Only a single genotype, GN-2085 gave the expression level of CrylAc $(1.31 \mu \mathrm{g} / \mathrm{g})$ that is near to the Environmental Protection Agency (EPA-USA) recommended dose (1.5 $\mu \mathrm{g} / \mathrm{g}$ ) while all other entries possessed toxin level well below $1.5 \mu \mathrm{g} / \mathrm{g}$ for durable resistance (Table 2).

Quantification of Bt toxin (Cry1Ac) level in 75 genotypes during 2012: Total of seventy-five genotypes were used for $B t$ gene(s) expression profiling in this year. All the genotypes were positive for CrylAc except DNH-105, BH-176 and CIM-573 (non Bt. Standard). The expression level for CrylAc in all the positive genotypes was measured in the range of 0.05-0.86 $\mu \mathrm{g} / \mathrm{g}$ which was low as compared to EPA-USA recommended toxin dose $(>1.5 \mu \mathrm{g} / \mathrm{g})$. JS-1, SB-149, SLH-4 and AA-904 gave the highest expression level of CrylAc $(0.86,0.82,0.81$ and $0.80 \mu \mathrm{g} / \mathrm{g}$, respectively), while PB-38, NIAB-112, GS-444, CIM-591, MPS-II and RCA-1 expressed minimum CrylAc expression level (0.05, 0.07, 0.07, 0.07, 0.07 and $0.08 \mu \mathrm{g} / \mathrm{g}$, respectively) (Table 3 ).

Quantification of Bt toxin (Cry1Ac) level in 49 genotypes during 2013: In a similar fashion of NCVT testing in 2013, forty-nine genotypes were used to find out the $B t$ toxin $($ CrylAc) level in candidate cotton genotypes. Among all the tested genotypes, CIM-573 (non Bt. Standard) and CRIS-342 (non Bt. Standard) were found negative for CrylAc expression. The expression level for the positive genotypes was measured in the range of 0.03-4.17 $\mu \mathrm{g} / \mathrm{g}$. Six genotypes, RCA-333, Tarzan-4, GH-142, SLH-4, VH-305 and AA-919 gave the highest CrylAc gene expression level (4.17, 3.96, $3.18,2.34,2.27$ and $2.06 \mu \mathrm{g} / \mathrm{g}$, respectively) that fulfils the 
EPA-USA requirement of $B t$ toxins for durable pest resistance $\quad 1$ showed the lowest expression level $(0.03,0.04,0.05$ and The genotypes MNH-988, Sahara-120, SLH-8 and NIAB-Bt. $\quad 0.09$ respectively) (Table 4).

Table 1. Immunostrip assay for local $B t$ cotton genotypes during the year 2009, 2012, 2013 and 2014.

(A) 2009

\begin{tabular}{|c|c|c|c|c|c|c|c|c|c|}
\hline Sr.\# & Entry & Cry1Ac & Cry2Ab & Cry $1 F$ & Sr.\# & Entry & Cry1Ac & Cry $2 A b$ & CrylF \\
\hline 1 & GN-31 & + & - & - & 7 & FH-113 & + & - & - \\
\hline 2 & GN-2085 & + & - & - & 8 & Ali Akbar-703 & + & - & - \\
\hline 3 & IR-3701 & + & - & - & 9 & CEMB-02 & + & - & - \\
\hline 4 & Ali Akbar-802 & + & - & - & 10 & Sitara-008 & + & - & - \\
\hline 5 & CEMB-01 & - & - & - & 11 & MG-6 & + & - & - \\
\hline 6 & IR-1524 & + & - & - & 12 & Neelam-121 & + & - & - \\
\hline \multicolumn{10}{|c|}{ (B) 2012} \\
\hline Sr.\# & Entry & Cry1Ac & Cry2Ab & Cry1F & Sr.\# & Entry & CrylAc & Cry $2 A b$ & CrylF \\
\hline 1 & HBC-SD-134 & + & - & - & 39 & FH-142 & + & - & - \\
\hline 2 & HBC-SB-814 & + & - & - & 40 & CEMB-33 & + & - & - \\
\hline 3 & HSP-3 & + & - & - & 41 & MNH-456 & + & - & - \\
\hline 4 & Kissan Early & + & + & - & 42 & VH-303 & + & - & - \\
\hline 5 & HSP-1 & + & - & - & 43 & BZU-75 & + & - & - \\
\hline 6 & Silver-White & + & - & - & 44 & VH-282 & + & - & - \\
\hline 7 & HSP-2 & + & - & - & 45 & CRIS-508 & + & - & - \\
\hline 8 & Silver-Gold & + & - & - & 46 & MM-58 & + & - & - \\
\hline 9 & AA-904 & + & - & - & 47 & IR-NIBGE-5 & + & - & - \\
\hline 10 & RS-1 & + & - & - & 48 & SLH-4 & + & - & - \\
\hline 11 & Auriga-213 & + & - & - & 49 & IUB-222 & + & - & - \\
\hline 12 & Tarzan-3 & + & - & - & 50 & IR-NIBGE-4 & + & - & - \\
\hline 13 & Sayban-201 & + & - & - & 51 & CIM-600 & + & - & - \\
\hline 14 & A-011 & + & - & - & 52 & ВН-180 & + & - & - \\
\hline 15 & TARZAN-2 & + & - & - & 53 & CIM-602 & + & - & - \\
\hline 16 & Sitara 10M & + & - & - & 54 & CEMB-55 & + & - & - \\
\hline 17 & SB-149 & + & - & - & 55 & CIM-599 & + & - & - \\
\hline 18 & Sitara-12 & + & - & - & 56 & CEMB-44 & + & - & - \\
\hline 19 & Sun-1 & + & - & - & 57 & Bt-BH-178 & + & - & - \\
\hline 20 & Sitara $11 \mathrm{M}$ & + & - & - & 58 & GS-444 & + & - & - \\
\hline 21 & KZ-389 & + & - & - & 59 & DNH-105 & - & - & - \\
\hline 22 & JS-1 & + & - & - & 60 & CIM-612 & + & - & - \\
\hline 23 & Leader-1 & + & - & - & 61 & PB-38 & + & - & - \\
\hline 24 & BGC-09 & + & - & - & 62 & IUB-11 & + & - & - \\
\hline 25 & Sayban-202 & + & - & - & 63 & MPS-II & + & - & - \\
\hline 26 & Silkee & + & - & - & 64 & NIAB-112 & + & - & - \\
\hline 27 & RCA-2 & + & - & - & 65 & Cyto-124 & + & + & - \\
\hline 28 & NS-161 & + & - & - & 66 & JS-212 & + & - & - \\
\hline 29 & AGC-777 & + & - & - & 67 & VH-300 & + & - & - \\
\hline 30 & AA-919 & + & - & - & 68 & CRIS-510 & + & - & - \\
\hline 31 & RCA-1 & + & - & - & 69 & NIA-80 & + & - & - \\
\hline 32 & BS-52 & + & - & - & 70 & CIM-591 & + & - & - \\
\hline 33 & Trend-1 & + & - & - & 71 & BH-176 & - & - & - \\
\hline 34 & A-555 & + & - & - & 72 & $\begin{array}{l}\text { CIM-573 (Non Bt } \\
\text { Standard) }\end{array}$ & - & - & - \\
\hline 35 & NIAB-Bt-1 & + & - & - & 73 & CRIS-342 (Standard) & + & - & - \\
\hline 36 & RH-627 & + & - & - & 74 & CIM-598 (Standard) & + & - & - \\
\hline 37 & FH-118 & + & - & - & 75 & MNH-886 (Standard) & + & - & - \\
\hline 38 & GH-142 & + & - & - & --- & --- & --- & --- & --- \\
\hline
\end{tabular}


(C) 2013

\begin{tabular}{|c|c|c|c|c|c|c|c|c|c|}
\hline Sr.\# & Entry & Cry1Ac & Cry $2 A b$ & CrylF & Sr.\# & Entry & Cry1Ac & Cry $2 A b$ & CrylF \\
\hline 1 & AA-919 & + & - & - & 26 & BZU-75 & + & - & - \\
\hline 2 & CA-926 & + & - & - & 27 & GH-142 & + & - & - \\
\hline 3 & BGC-09 & + & - & - & 28 & MM-58 & + & - & - \\
\hline 4 & Syban-202 & + & - & - & 29 & IUB-13 & + & - & - \\
\hline 5 & BS-52 & + & - & - & 30 & VH-303 & + & - & - \\
\hline 6 & Trend-1 & + & - & - & 31 & VH-305 & + & - & - \\
\hline 7 & Leader-1 & + & - & - & 32 & CIM-600 & + & - & - \\
\hline 8 & Leader-5 & + & - & - & 33 & CIM-616 & + & - & - \\
\hline 9 & Sun-1 & + & - & - & 34 & Cyto-177 & + & - & - \\
\hline 10 & Leader-3 & + & - & - & 35 & ВН-180 & + & - & - \\
\hline 11 & Al-Seemi H Bt. 209 & + & - & - & 36 & BH-184 & + & - & - \\
\hline 12 & JS-1 & + & - & - & 37 & SLH-4 & + & - & - \\
\hline 13 & Sitara-12 & + & - & - & 38 & SLH-8 & + & - & - \\
\hline 14 & Sitara-13 & + & - & - & 39 & FH-142 & + & - & - \\
\hline 15 & AGC-777 & + & - & - & 40 & FH-Lalazar & + & - & - \\
\hline 16 & AGC-999 & + & - & - & 41 & IR-NIBGE-5 & + & - & - \\
\hline 17 & Tarzan-3 & + & - & - & 42 & IR-NIBGE-6 & + & - & - \\
\hline 18 & Tarzan-4 & + & - & - & 43 & CIM-598 (Bt. Standard) & + & - & - \\
\hline 19 & Eagle-1 & + & - & - & 44 & MNH-886 (Bt. Standard) & + & - & - \\
\hline 20 & HS-81213 & + & - & - & 45 & MNH-988 & + & - & - \\
\hline 21 & RCA-333 & + & - & - & 46 & CIM-573 (non Bt. Standard) & - & - & - \\
\hline 22 & LS-62 & + & - & - & 47 & $\begin{array}{l}\text { CRIS-342 (non Bt. } \\
\text { Standard) }\end{array}$ & - & - & - \\
\hline 23 & Sahara-120 & + & - & - & 48 & CEMB-55 & + & - & - \\
\hline 24 & NIAB-Bt. 1 & + & - & - & 49 & CEMB-66 & + & - & - \\
\hline 25 & RH-627 & + & - & - & --- & --- & --- & --- & --- \\
\hline \multicolumn{10}{|c|}{ (D) 2014} \\
\hline Sr.\# & Entry & CrylAc & Cry $2 A b$ & Cry1F & Sr.\# & Entry & Cry1Ac & Cry $2 A b$ & Cry1F \\
\hline 1 & Baghdadi & + & - & - & 23 & CRIS-342 (non Bt. Standard) & + & - & - \\
\hline 2 & CEMB-77 & + & - & - & 24 & CIM-573 (non Bt. Standard) & + & - & - \\
\hline 3 & CIM-622 & + & - & - & 25 & FH-142 (Bt. Standard-2) & + & - & - \\
\hline 4 & Cyto-178 & + & - & - & 26 & CIM-602 (Bt. Standard -1) & + & - & - \\
\hline 5 & IR-NIBGE-7 & + & - & - & 27 & Bt-Hybrid-53 & + & - & - \\
\hline 6 & BH-185 & - & - & - & 28 & Al Seemi H Bt. 209 & + & - & - \\
\hline 7 & FH-Noor & + & - & - & 29 & Tahafuz-3 & + & - & - \\
\hline 8 & VH-327 & + & - & - & 30 & BS-70 & + & - & - \\
\hline 9 & NIAB-874B & + & - & - & 31 & CRYSTAL-1 & + & - & - \\
\hline 10 & RH-647 & + & - & - & 32 & JS-733 & + & - & - \\
\hline 11 & TH-21/09 & + & - & - & 33 & SAHARA-150 & + & - & - \\
\hline 12 & IUB 63 & + & - & - & 34 & AGC-Nazeer-1 & + & - & - \\
\hline 13 & IUB-13 & + & - & - & 35 & Sitara-14 & + & - & - \\
\hline 14 & CEMB-66 & + & - & - & 36 & Auriga-215 & + & - & - \\
\hline 15 & IR-NIBGE-6 & + & - & - & 37 & SAHARA-120 & + & - & - \\
\hline 16 & FH-Lalazar & + & - & - & 38 & Eagle-1 & + & - & - \\
\hline 17 & MNH-988 & + & - & - & 39 & Tarzan-4 & + & - & - \\
\hline 18 & VH-305 & + & - & - & 40 & AGC-999 & + & - & - \\
\hline 19 & SLH-8 & - & - & - & 41 & Sitara-13 & + & - & - \\
\hline 20 & BH-184 & + & - & - & 42 & CA-926 & + & - & - \\
\hline 21 & Cyto-177 & + & - & - & 43 & Leader-3 & + & - & - \\
\hline 22 & CIM-616 & + & - & - & 44 & Leader-5 & + & - & - \\
\hline
\end{tabular}

$(+)=B t$ gene presence; $(-)=B t$ gene absence 
Table 2. Bt toxin (Cry1Ac) level for 12 cotton genotypes during 2009.

\begin{tabular}{llcllc}
\hline Sr. \# & Genotype/Entry & $\begin{array}{c}\text { Cry1Ac } \text { Toxin level } \\
(\boldsymbol{\mu g} / \mathbf{g}) \text { at 80 DAS }\end{array}$ & Sr. \# & Genotype/Entry & $\begin{array}{c}\text { Cry1Ac Toxin level } \\
(\boldsymbol{\mu g} / \mathbf{g}) \text { at 80 DAS }\end{array}$ \\
\hline 1 & GN-31 & 0.88 & 7 & FH-113 & 0.21 \\
2 & GN-2085 & 1.31 & 8 & Ali Akbar-703 & 0.41 \\
3 & IR-3701 & 0.25 & 9 & CEMB-02 & 0.48 \\
4 & Ali Akbar-802 & 0.45 & 10 & Sitara-008 & 0.30 \\
5 & CEMB-01 & 0.00 & 11 & MG-6 & 0.27 \\
6 & IR-1524 & 0.31 & 12 & Neelam-121 & 0.22 \\
\hline
\end{tabular}

Table 3. Bt toxin (Cry1Ac) level for 75 cotton genotypes during 2012.

\begin{tabular}{|c|c|c|c|c|c|}
\hline Sr.\# & Genotype/Entry & $\begin{array}{c}\text { Cry1Ac Toxin level } \\
(\mu \mathrm{g} / \mathrm{g}) \text { 80 DAS }\end{array}$ & Sr.\# & Genotype/Entry & $\begin{array}{c}\text { Cry1Ac Toxin level } \\
(\mu \mathrm{g} / \mathrm{g}) \text { 80 DAS }\end{array}$ \\
\hline 1 & HBC-SD-134 & 0.63 & 39 & FH-142 & 0.61 \\
\hline 2 & HBC-SB-814 & 0.67 & 40 & CEMB-33 & 0.63 \\
\hline 3 & HSP-3 & 0.52 & 41 & MNH-456 & 0.37 \\
\hline 4 & Kissan Early & 0.48 & 42 & VH-303 & 0.60 \\
\hline 5 & HSP-1 & 0.57 & 43 & BZU-75 & 0.50 \\
\hline 6 & Silver-White & 0.48 & 44 & VH-282 & 0.62 \\
\hline 7 & HSP-2 & 0.54 & 45 & CRIS-508 & 0.73 \\
\hline 8 & Silver-Gold & 0.58 & 46 & MM-58 & 0.46 \\
\hline 9 & AA-904 & 0.80 & 47 & IR-NIBGE-5 & 0.39 \\
\hline 10 & RS-1 & 0.70 & 48 & SLH-4 & 0.81 \\
\hline 11 & Auriga-213 & 0.76 & 49 & IUB-222 & 0.33 \\
\hline 12 & Tarzan-3 & 0.32 & 50 & IR-NIBGE-4 & 0.39 \\
\hline 13 & Sayban-201 & 0.33 & 51 & CIM-600 & 0.64 \\
\hline 14 & A-011 & 0.53 & 52 & BH-180 & 0.48 \\
\hline 15 & TARZAN-2 & 0.79 & 53 & CIM-602 & 0.51 \\
\hline 16 & Sitara 10M & 0.46 & 54 & CEMB-55 & 0.63 \\
\hline 17 & SB-149 & 0.82 & 55 & CIM-599 & 0.22 \\
\hline 18 & Sitara-12 & 0.46 & 56 & CEMB-44 & 0.39 \\
\hline 19 & Sun-1 & 0.73 & 57 & Bt-BH-178 & 0.24 \\
\hline 20 & Sitara $11 \mathrm{M}$ & 0.39 & 58 & GS-444 & 0.07 \\
\hline 21 & KZ-389 & 0.62 & 59 & DNH-105 & 0 \\
\hline 22 & JS-1 & 0.86 & 60 & CIM-612 & 0.09 \\
\hline 23 & Leader-1 & 0.41 & 61 & PB-38 & 0.05 \\
\hline 24 & BGC-09 & 0.70 & 62 & IUB-11 & 0.76 \\
\hline 25 & Sayban-202 & 0.68 & 63 & MPS-II & 0.07 \\
\hline 26 & Silkee & 0.46 & 64 & NIAB-112 & 0.07 \\
\hline 27 & RCA-2 & 0.72 & 65 & Cyto-124 & 0.72 \\
\hline 28 & NS-161 & 0.53 & 66 & JS-212 & 0.58 \\
\hline 29 & AGC-777 & 0.58 & 67 & VH-300 & 0.57 \\
\hline 30 & AA-919 & 0.29 & 68 & CRIS-510 & 0.61 \\
\hline 31 & RCA-1 & 0.08 & 69 & NIA-80 & 0.77 \\
\hline 32 & BS-52 & 0.44 & 70 & CIM-591 & 0.07 \\
\hline 33 & Trend-1 & 0.36 & 71 & BH-176 & 0 \\
\hline 34 & A-555 & 0.31 & 72 & $\begin{array}{l}\text { CIM-573 (non Bt } \\
\text { Standard) }\end{array}$ & 0 \\
\hline 35 & NIAB-Bt-1 & 0.39 & 73 & CRIS-342 (Standard) & 0.09 \\
\hline 36 & RH-627 & 0.42 & 74 & CIM-598 (Standard) & 0.28 \\
\hline 37 & FH-118 & 0.65 & 75 & MNH-886 (Standard) & 0.59 \\
\hline 38 & GH-142 & 0.41 & --- & --- & ---- \\
\hline
\end{tabular}


Table 4. Bt toxin (Cry1Ac) level for 49 cotton genotypes during 2013.

\begin{tabular}{llcclc}
\hline Sr.\# & Genotype/Entry & $\begin{array}{c}\text { Cry1Ac Toxin level } \\
(\boldsymbol{\mu g} / \mathbf{g}) \text { at 80 DAS }\end{array}$ & Sr.\# & Genotype/Entry & $\begin{array}{c}\text { Cry1Ac Toxin level } \\
(\boldsymbol{\mu g} / \mathbf{g}) \text { at 80 DAS }\end{array}$ \\
\hline 1 & AA-919 & 2.06 & 26 & BZU-75 & 0.23 \\
2 & CA-926 & 1.00 & 27 & GH-142 & 3.18 \\
3 & BGC-09 & 0.18 & 28 & MM-58 & 0.86 \\
4 & Syban-202 & 0.98 & 29 & IUB-13 & 0.24 \\
5 & BS-52 & 0.65 & 30 & VH-303 & 0.16 \\
6 & Trend-1 & 1.33 & 31 & VH-305 & 2.27 \\
7 & Leader-1 & 0.41 & 32 & CIM-600 & 0.90 \\
8 & Leader-5 & 0.50 & 33 & CIM-616 & 0.75 \\
9 & Sun-1 & 0.11 & 34 & Cyto-177 & 0.20 \\
10 & Leader-3 & 0.51 & 35 & BH-180 & 0.98 \\
11 & Al-Seemi H Bt. 209 & 0.78 & 36 & BH-184 & 0.08 \\
12 & JS-1 & 0.59 & 37 & SLH-4 & 2.34 \\
13 & Sitara-12 & 0.24 & 38 & SLH-8 & 0.05 \\
14 & Sitara-13 & 0.65 & 39 & FH-142 & 1.33 \\
15 & AGC-777 & 0.60 & 40 & FH-Lalazar & 0.14 \\
16 & AGC-999 & 0.28 & 41 & IR-NIBGE-5 & 0.23 \\
17 & Tarzan-3 & 0.15 & 42 & IR-NIBGE-6 & 0.21 \\
18 & Tarzan-4 & 3.96 & 43 & CIM-598 (Bt. Standard) & 1.25 \\
19 & Eagle-1 & 0.27 & 44 & MNH-886 (Bt. Standard) & 0.13 \\
20 & HS-81213 & 0.43 & 45 & MNH-988 & 0.03 \\
21 & RCA-333 & 4.17 & 46 & CIM-573 (non Bt. Standard) & 0.00 \\
22 & LS-62 & 0.15 & 47 & CRIS-342 (non Bt. Standard) & 0.00 \\
23 & Sahara-120 & 0.04 & 48 & CEMB-55 & 0.11 \\
24 & NIAB-Bt. 1 & 0.09 & 49 & CEMB-66 & 0.39 \\
25 & RH-627 & 0.20 & --- & --- & ---- \\
\hline
\end{tabular}

Table 5. Bt toxin (Cry1Ac) level for 44 cotton genotypes during 2014.

\begin{tabular}{llcclc}
\hline Sr.\# & Genotype/Entry & $\begin{array}{c}\text { Cry1Ac } \text { Toxin level } \\
(\boldsymbol{\mu g} / \mathbf{g}) \text { at 80 DAS }\end{array}$ & Sr.\# & Genotype/Entry & $\begin{array}{c}\text { Cry1Ac } \text { Toxin level } \\
(\boldsymbol{\mu g} / \mathbf{g}) \text { at 80 DAS }\end{array}$ \\
\hline 1 & Baghdadi & 1.22 & 23 & CRIS-342 (non Bt. Standard) & 0 \\
2 & CEMB-77 & 0.74 & 24 & CIM-573 (non Bt. Standard) & 0.42 \\
3 & CIM-622 & 2.38 & 25 & FH-142 (Bt. Standard-2) & 1.52 \\
4 & Cyto-178 & 0.25 & 26 & CIM-602 (Bt. Standard-1) & 0.17 \\
5 & IR-NIBGE-7 & 0.54 & 27 & Bt-Hybrid-53 & 2.48 \\
6 & BH-185 & 0.00 & 28 & Al Seemi H Bt. 209 & 0.91 \\
7 & FH-Noor & 0.25 & 29 & Tahafuz-3 & 1.69 \\
8 & VH-327 & 0.17 & 30 & BS-70 & 0.44 \\
9 & NIAB-874B & 1.41 & 31 & CRYSTAL-1 & 0.37 \\
10 & RH-647 & 1.61 & 32 & JS-733 & 0.54 \\
11 & TH-21/09 & 3.90 & 33 & SAHARA-150 & 0.16 \\
12 & IUB-63 & 0.09 & 34 & AGC-Nazeer-1 & 0.70 \\
13 & IUB-13 & 2.12 & 35 & Sitara-14 & 0.59 \\
14 & CEMB-66 & 2.02 & 36 & Auriga-215 & 0.72 \\
15 & IR-NIBGE-6 & 1.96 & 37 & SAHARA-120 & 1.68 \\
16 & FH-Lalazar & 1.76 & 38 & Eagle-1 & 0.50 \\
17 & MNH-988 & 0.93 & 39 & Tarzan-4 & 4.29 \\
18 & VH-305 & 0.05 & 40 & AGC-999 & 0.24 \\
19 & SLH-8 & 0.00 & 41 & Sitara-13 & 0.11 \\
20 & BH-184 & 1.10 & 42 & CA-926 & 3.42 \\
21 & Cyto-177 & 1.10 & 43 & Leader-3 & 2.26 \\
22 & CIM-616 & 0.12 & 44 & Leader-5 & 1.72 \\
\hline
\end{tabular}


Quantification of Bt toxin (Cry1Ac) level in 44 genotypes during 2014: Forty-four genotypes of NCVT were assessed during 2014 to find CrylAc expression level in candidate cotton genotypes. Results revealed that only 3 genotypes BH-185, SLH-8 and CRIS-342 (non Bt. Standard) were negative, while rest of all the genotypes were positive for the expression level of CrylAc. The toxin level for all positive genotypes was measured in the range of 0.05-4.29 $\mu \mathrm{g} / \mathrm{g}$. The maximum expression level for CrylAc was observed in Tarzan-4, TH-21/09, CA-926 and Bt-Hybrid-53 (4.29, 3.90, 3.42 and $2.48 \mu \mathrm{g} / \mathrm{g}$ respectively). However, the lowest expression level was recorded in the genotypes VH-305, IUB 63, Sitara-13 and CIM-616 (0.05, 0.09, 0.11 and 0.12 respectively) (Table 5). It means that the expression level of CrylAc gene was increased gradually up to the limit of EPAUSA due to the fact that true breeder seeds of transgenic cotton were crossed with approved standards of CrylAc gene (Mon-531 event).

\section{DISCUSSION}

One of the main consequences of $B t$ cotton technology raised due to the gradual reduction in toxin level and as result of fact, the expected development of insect resistance against $B t$ toxin is a major concern in developing countries like Pakistan. There are various factors that affect the efficacy of $B t$ toxin level (mainly abiotic stresses) due to which final toxin quantity is altered (Mahon et al., 2002). Still so far, in Pakistan no base line of $B t$ toxin has been recommended due to lack of proper implementation and coordination among the concerned institutions. Keeping in view the standard toxin level $1.5 \mu \mathrm{g} / \mathrm{g}$ of EPA-USA and $1.8 \mu \mathrm{g} / \mathrm{g}$ (Kranthi et al., 2005), this study was conducted during the successive years (2009, 2012, 2013, and 2014) to confirm Bt gene type and simultaneously quantify the level of toxin of 180 cotton genotypes at 80 days after sowing of NCVT.

During first year of experimentation (2009), a total of twelve genotypes were assessed for the expression profiling of $B t$ gene $($ CrylAc) and data fall in the range of $0.21-1.31 \mu \mathrm{g} / \mathrm{g}$. The toxin level was very low as compared to international standards of EPA-USA $(1.5 \mu \mathrm{g} / \mathrm{g})$. This lower level of expression was mainly due to the reason that quantified level of $B t$ toxin was not given consideration during the breeding of these varieties. Another clear reason was the origin of genetic backgrounds. For example, GN-31 and GN-2085 belonged to Indian origin possessed much better level of expression compared to all other locally bred cotton varieties. GN-2085 was later approved for commercial cultivation, but it could not be realized at farmer level due to seed availability. It was hybrid in nature and Pakistan cotton seed import rule prohibited its import in bulk ( $>5 \mathrm{Ibs}$.) The expression exhibited by the locally bred genotypes in the year 2009 was not satisfactory keeping in view the international standards. For durable protection against specific insect pests, appropriate level of toxin is important at specific stage and time. Other findings by Kranthi et al. (2005), demonstrated that for durable pest resistance, $B t$ toxin $1.8 \mu \mathrm{g} / \mathrm{g}$ fresh weight leaf tissue basis or higher is recommended. This value is slightly higher than EPA-USA recommended dose of $B t$ toxin and it may possibly be due to the differences in plant genotypes and local environmental conditions that affect the Cry genes expression.

Regarding, the results of NCVT in 2012, seventy-five genotypes were analyzed to find out the expression level of $B t$ gene. Overall data of 75 genotypes during 2012, gave the expression level in the range of $0.05-0.86 \mu \mathrm{g} / \mathrm{g}$. The situation was still alarming. The expression trend in this year was very much similar to the year 2009 and was well below the international reported level for durable resistance (Kranthi et al., 2005). Probably, cotton lines tested in this year were developed in similar fashion (least concern for toxin level) like the line of year 2009, so not much variation could be expected still. According to Mahon et al. (2002), variation below certain level in $B t$ protein may decrease the efficacy of $B t$ cotton to control target worms. Appropriate level of $B t$ toxin play an important role in plant protection against target pest. Another reason for this low expression was that proper host genetic backgrounds could not be identified still to attain appropriate level or improve the existing levels of toxin by the local plant breeders in the country.

The expression level for $B t$ gene during 2013 was recorded in the range of $0.03-4.17 \mu \mathrm{g} / \mathrm{g}$ for forty-nine genotypes of NCVT. Surprisingly during this year, six genotypes gave their best expression that was more than the international recommended values. In 2014, NCVT was designed to screen the expression level of forty-four genotypes. All the genotypes under experimentation gave the expression range of $0.05-4.29 \mu \mathrm{g} / \mathrm{g}$. In this study fifteen tested genotypes gave the best expression in the range of $1.52-4.29 \mu \mathrm{g} / \mathrm{g}$. The results of these two years (2013 \& 2014) revealed that CrylAc expression level in the twenty tested genotypes was found to be higher than the expression level of EPA-USA $(1.5 \mu \mathrm{g} / \mathrm{g})$ for durable insect pest resistance. So, there seemed to be improvement in the toxin levels during the years 2013 and 2014 compared to previous years data of 2009 and 2012. The consistent improvement in the toxin level during the years 2013 and 2014 is a positive sign in the cotton research of Pakistan. The cotton breeders might have undertaken the parameter of improved toxin level in their breeding programs and further consistent improvement can be expected in the coming years.

Conclusion: The overall picture of four years data depicts that toxin level in the breeder seed seemed to be improved in terms of $1^{\text {st }}$ generation $B t$ toxin level and other traits as a result of better selections. The possible reasons for this improvement may be improved genetic backgrounds, better genetic response to inputs and tolerance to abiotic stresses 
(heat, salinity and drought). Furthermore, Bt genes expression in the approved cotton varieties need to be continuously monitored during the crop growing season and over the years according to standards. A threshold level of $B t$ toxin is very crucial as extremely low level of toxin may lead to the development of cross resistance. There is also need to introduce $2^{\text {nd }}$ and $3^{\text {rd }}$ generation insect resistant transgenic cotton varieties to get diversification of $\mathrm{Bt}$ genes, and release the building pressure of developing cross resistance over the existing $1^{\text {st }}$ generation $B t$ cotton technology. It is need of time to develop awareness among the farmers regarding the appropriate management practices for fully utilizing the $B t$ potential taking into account the ineffectiveness of $B t$ against sucking insects pests which require conventional pest management measures.

\section{REFERENCES}

Adamczyk, J.J. and D.V. Sumerford. 2001. Potential factors impacting season-long expression of CrylAc in 13 commercial varieties of Bollgard $\AA$ cotton. J. Insect Sci.1:1-6.

Ali, S., S. Hameed, S. Masood, G.M. Ali and Y. Zafar. 2010. Status of $B t$ cotton cultivation in major growing areas of Pakistan. Pak. J. Bot. 42:1583-1594.

Alstad, D.N. and D.A. Andow. 1995. Managing the evolution of resistance to transgenic plants. Science. 268:1894 -1896.

Barrett-Lennard, E.G. 2003. The interaction between waterlogging and salinity in higher plants: causes, consequences and implications. Plant Soil. 253: 35-54.

Benedict, J.H., E.S. Sachs, D.W. Altman, W.R. Deaton, R.J. Kohel, D.R. Ring and S.A. Berberich. 1996. Field performance of cottons expressing transgenic CrylA insecticidal proteins for resistance to Heliothis virescens and Helicoverpa zea (Lepidoptera: Noctuidae). J. Econ. Entomol. 89:230-238.

Chen, D., G. Ye, C. Yang, Y. Chen and Y. Wu. 2005. The effect of high temperature on the insecticidal properties of Bt cotton. Environ. Exp. Bot. 53:333-342.

Coviella, C.E., D.J. Morgan and J.T. Trumble. 2000. Interactions of elevated $\mathrm{CO}_{2}$ and nitrogen fertilization: Effects on production of Bacillus thuringiensis toxins in transgenic plants. Environ. Entomol. 29:781-787.

Coviella, C.E., R.D. Stipanovic and J.T. Trumble. 2002. Plant allocation to defensive compounds: interactions between elevated $\mathrm{CO}_{2}$ and nitrogen in transgenic cotton plants. J. Exp. Bot. 53: 323-331.

Gutierrez, A.P., J.J. Adamczyk Jr, S. Ponsard and C.K. Ellis. 2006. Physiologically based demographics of $B t$ cottonpest interactions: II. Temporal refuges, natural enemy interactions. Eco. Model. 191: 360-382.
Kranthi, K.R., S. Naidu, C.S. Dhawad, A. Tatwawadi, K. Mate, E. Patil, and S. Kranthi. 2005. Temporal and intraplant variability of CrylAc expression in Bt cotton and its influence on the survival of the cotton bollworm, Helicoverpa armigera (Hubner) (Noctuidae: Lepidoptera). Curr. Sci. 89: 291-297.

Liang, G.M., W.J. Tan and Y.Y. Guo. 2000. Study on screening and inheritance mode of resistance to $\mathrm{Bt}$ transgenic cotton in cotton bollworm. Acta Entomol. Sin. 43:57-43.

Mahon, R., J. Finnergan, K. Olsen and L. Lawrence. 2002. Environmental stress and the efficacy of Bt cotton. Aust. Cotton Grower 23:18-21.

McGaughey, W.H. and M.E. Whalon. 1992. Managing insect resistance to Bacillus thuringiensis toxins. Science. 258:1451-1455.

Olsen, K.M., J.C Daly, H.E. Holt and E.J Finnegan 2005. Season-long variation in expression of CrylAc gene and efficacy of Bacillus thuringiensis toxin in transgenic cotton against Helicoverpa armigera (Lepidoptera: Noctuidae). J. Econ. Entomol. 98:1007-1017.

Vasquez, O. and M.S.U. Rehman. 2013. Pakistan Agriculture Biotechnology Annual. Available online at http://gain.fas.usda.gov/Recent\%20GAIN\%20Publicati ons/Agricultural\%20Biotechnology\%20Annual_Islama bad_Pakistan_7-18-2013.pdf

Peck, S.L., F. Gould and S.P Ellner. 1999. Spread of resistance in spatially extended regions of transgenic cotton: implications for management of Heliothis virescens (Lepidoptera: Noctuidae). J. Econ. Entomol. 92:1-16.

Pray, C.E., D. Ma, J. Huang and F. Qiao. 2001. Impact of $B t$ cotton in China. World Dev. 29:813-825.

Tabashnik, B.E. 1994. Evolution of resistance to Bacillus thuringiensis. Annu. Rev. Entomol. 39:47-79.

Tabashnik, B.E., A.J. Gassmann, D.W. Crowder and Y. Carriere. 2008. Insect resistance to $B t$ crops: evidence versus theory. Nat. Biotechnol. 26:199-202.

$\mathrm{Wu}, \mathrm{K}$. and Y. Guo. 1997. The influence of gene flow between geographical populations on the evolution of insecticide resistance in Helicoverpa armigera. Acta Entomol. Sin. 40:30-34.

Wu, K., Y. Guo and N. Lv. 1999. Geographic variation in susceptibility of Bacillus thuringiensis insecticidal protein in China. J. Econ. Entomol. 92:273-278.

Wu, K.M. and Y.Y. Guo. 2005. The evolution of cotton pest management practices in China. Annu. Rev. Entomol. 50: 31-52.

Iqbal, A., S. Ali, M.A. Zia, A. Shahzad, J.U. Din, M.A. Ullah, G.M. Ali and Y. Zafar 2013. Comparative account of $\mathrm{Bt}$ gene expression in cotton under normal and salt affected soil. Int. J. Agric. Biol. 15:1181-1186. 